\title{
VIII. Internationale politische Aktivitäten
}

Neben die Organisation der Flüchtlingshilfe für die nach Kriegsende in Deutschland befindlichen DPs trat als zusätzliches Problem bald die Fluchtwelle, die mit der Machtübernahme der Kommunisten in den osteuropäischen Ländern in der zweiten Hälfte der vierziger Jahre eingesetzt hatte. Im Kontrast zu dieser Fluchtbewegung standen die Zwangsrepatriierungen eines Teils der DPs aus der Sowjetunion und den baltischen Staaten, die in die Mühlen stalinistischer Vergeltungsmaßnahmen geraten waren ${ }^{1}$. Die Flüchtlingsorganisationen und die ICMC wurden hier mit zahlreichen menschlichen Dramen konfrontiert, Schauff überdies mit der beängstigenden Analogie menschenverachtenden Verhaltens totalitärer Staaten, das er nach 1933 am eigenen Leibe erfahren hatte.

Bei seinen Bemühungen, Auswanderungsmöglichkeiten nach Lateinamerika zu erschließen, war er von daher auch sensibilisiert gegenüber antidemokratischen Entwicklungen in den dortigen Aufnahmeländern. Er war aber auch sensibilisiert und in Sorge um die politische Entwicklung und Orientierung der noch jungen Bundesrepublik Deutschland.

\section{Im Kreis der konservativen Remigration}

Schauff hatte, wie schon angeführt, im deutschen Weststaat bzw. in der jungen Bundesrepublik Deutschland kein neues Amt angestrebt. Nichtsdestoweniger stellte er seine Aktivitäten in Genf, einem der wichtigsten Orte internationaler Politik, umgehend auch in den Dienst der außenpolitisch noch keineswegs souveränen Bundesrepublik. Laut eigener Aussage war er aufgrund seiner internationalen Verbindungen zumindest am Rande daran beteiligt, auf Schweizer Boden die ersten Treffen Adenauers mit dem damaligen französischen Außenminister Georges Bidault vorzubereiten².

Diese Bemühungen galten der deutsch-französischen Verständigung und standen zugleich in Zusammenhang mit den seit 1947 in Genf stattfindenden $\mathrm{Zu}$ sammenkünften führender christlich-demokratischer Politiker aus Westeuropa. Solche Treffen waren von Jakob Kindt-Kiefer initiiert worden, der 1946 mit

1 Die Repatriierung ausnahmslos aller sowjetischen Staatsangehörigen war bereits auf der Konferenz von Jalta beschlossen und bis Ende 1945 auch weitgehend durchgeführt worden $(2034000$ Menschen $=98 \%)$. Die Zwangsrepatriierung betraf vor allem auch Angehörige der "Wlassow-Armee“ und der Kosaken-Verbände, die bereits seit dem russischen Bürgerkrieg im Exil gelebt hatten. Daneben waren es Esten, Letten, Balten und Ukrainer, die im Verdacht standen, mit den Deutschen kollaboriert zu haben. Zu dem sowjetischen Vorgehen und den Vergeltungsmaßnahmen vgl. Bethell, Das letzte Geheimnis; Jacobmeyer, Vom Zwangsarbeiter zum heimatlosen Ausländer, S. $123 \mathrm{ff}$.

2 Schauff, Autobiographische Notizen/Fragmente. Zu den damaligen streng vertraulichen „Genfer Gesprächen“" vgl. Schwarz, Adenauer. Der Aufstieg, S. 558 ff. 
Joseph Wirth die „Vereinigung Christlich-Demokratisches Deutschland in der Schweiz" gegründet hatte und später in der Saar-Politik der Bundesrepublik aktiv wurde ${ }^{3}$. Auch Schauffs Auslandsreisen als Repräsentant der ICMC, vor allem nach Lateinamerika und in die USA, standen immer zugleich im Dienst des politisch-gesellschaftlichen und wirtschaftlichen Aufbaus in Deutschland.

In den USA traf Schauff auf einen Kreis von Emigranten des konservativchristlichen Lagers, die zum Teil in einflußreiche Stellungen gelangt waren und nicht nach Deutschland zurückkehrten. Ein wichtiges Forum für deutschlandpolitische Aktivitäten von Emigranten war der 1951 von Christopher Emmet ins Leben gerufene "American Council on Germany“. Als überzeugter Christ bekämpfte Emmet die totalitäre Herrschaft des Sowjetkommunismus ebenso, wie er vor 1945 das nationalsozialistische Deutschland bekämpft hatte. Überzeugt von der Existenz eines „besseren Deutschland“ und Gegner der KollektivschuldThese, wandte er sich schon unmittelbar nach dem Krieg gegen die Demontage der deutschen Industrie und die Realisierung der Morgenthau-Ideen, die auch in der amerikanischen Besatzungsdirektive JCS 1067 noch deutliche Spuren hinterlassen hatten ${ }^{5}$. Dem American Council on Germany präsidierten so bedeutende Amerikaner wie der Hohe Kommissar für Deutschland, John $\mathrm{McCloy}^{6}$, und General Lucius D. Clay, bis 1949 Militärgouverneur der amerikanischen Besatzungszone und Organisator der Berliner Luftbrücke; dem weiteren Präsidium und Board gehörten mehr als ein Drittel Emigranten und Remigranten konservativer Provenienz an, darunter so bekannte Namen wie Eric Warburg ${ }^{7}$, Karl Brandt ${ }^{8}$, der ehemalige Chefredakteur des "Christlichen Ständestaat" und Schauff-Intimus Klaus Dohrn ${ }^{9}$ sowie Hajo Holborn ${ }^{10}$, Hans Staudinger ${ }^{11}$ und Norbert Mueh-

3 Jakob Kindt-Kiefer war 1935 in die Schweiz emigriert, April 1945 mit Otto Braun und Wilhelm Hoegner Begründer der Arbeitsgemeinschaft „Das demokratische Deutschland", engagierter Föderalist, Mithrsg. der „Richtlinien für ein demokratisches, republikanisches, föderalistisches Deutschland“ vom Mai 1945 (BHB I). Zu den frühen christdemokratischen Treffen vgl. Dörpinghaus, Genfer Sitzungen.

4 Christopher T. Emmet (1900-1974), amerikanischer Publizist, vor 1933 Studium u.a. in Deutschland (Nachlaß Christopher Temple Emmet in Hoover Institution, Stanford; vgl. auch Nachlaß Marcia Kahn, IfZ, ED 364).

5 Der amerikanische Finanzminister Henry Morgenthau hatte - neben der weitgehenden Zerstückelung und Internationalisierung - die Entindustrialisierung bzw. „Agrarisierung “ Deutschlands gefordert. Zum Einfluß Morgenthaus auf die amerikanische Besatzungsdirektive JCS 1067 vgl. Henke, Amerikanische Besetzung, S. $107 \mathrm{ff}$.

6 John McCloy war 1949-1952 Hoher Kommissar für Deutschland.

7 Eric Warburg (1900-1990), Bankier; emigrierte 1938 in die USA, im Zweiten Weltkrieg US-Nachrichtendienst, Mitarbeiter der "Association for a Democratic Germany" und Mitglied des „Hilfskomitees für Überlebende deutscher Widerstandskämpfer“; 1956 Rückkehr nach Deutschland, Bankier in Hamburg, beteiligt am Wiederaufbau der dortigen jüdischen Gemeinde (BHB I).

8 Karl Brandt (1899-1975), Agrarwissenschaftler, Hochschullehrer; aktiver NS-Gegner, 1933 Emigration in die USA, Professor an der New School for Social Research, 1938-1963 an der Stanford Universität, während des Krieges Regierungsberater, nach 1945 Berater von General Clay und OMGUS, ab 1948 mehrfach Gastprofessor an westdeutschen Universitäten (BHB II).

9 Klaus Dohrn (1913-1979), 1933 Mitarbeiter und Chefredakteur „Der Christliche Ständestaat" in Wien, war 1937 Mitbegründer der „Deutschen Front gegen das Hitlerregime“ als 
len ${ }^{12}$; weitere beratende Mitglieder und Mitarbeiter waren u.a. Alexander Böker, der bei Heinrich Brüning in Harvard promoviert hatte ${ }^{13}$, und nicht zuletzt Johannes Schauff. Der Council, der beträchtliche Einflußmöglichkeiten in amerikanischen Regierungskreisen besaß, setzte sich für eine wirtschaftlichen Aufbauhilfe für Deutschland und für eine Stärkung der politischen Eigenständigkeit der Bevölkerung ein; zudem sollten die „Morgenthau und Vansittart boys“ so schnell wie möglich abgelöst werden, „simply jobholders who live off the land, ... a constant source of irritation of the German people ..." 14 .

Zusammenschluß deutscher konservativer Exilgruppen in Österreich und der CSR; er floh 1938 in die CSR, 1939 nach Paris, war aktiv in der konservativen österreichischen Exilpolitik; 1941 über Südfrankreich und Spanien Flucht nach Portugal, von dort 1942 in die USA, Mitarbeiter in katholischen Hilfsorganisationen, nach dem Kriege europäischer Berater von Henry Luce für die Magazine Time und Life (BHB I); die ausführliche Korrespondenz Schauff-Dohrn 1956-1976 in: IfZ, NL Schauff, Bd. 2 u. 32.

10 Hajo Holborn (1902-1969), Historiker und Hochschullehrer, 1931 Carnegie-Professor an der Deutschen Hochschule für Politik, 1933 Emigration in die USA, 1934-1969 Professor an der Yale University; Holborn hatte in NSDAP-Kreisen als "weit linksstehend" gegolten (vgl. Heiber, Karl Frank, S. 169), wird jedoch von Radkau dem „Emigranten-Konservativismus" zugerechnet (vgl. Radkau, Deutsche Emigration, S. 299). Er war während des Krieges Mitarbeiter und schließlich Chef der Research and Analyst Branch des OSS, 1947-1949 Berater des Außenministeriums; nahm 1960 leitende Funktionen im American Council on Germany wahr. Er beeinflußte in seiner hohen OSS-Funktion die amerikanische Deutschlandpolitik im Sinne der These, daß der Nationalsozialismus keine logische Folge der deutschen Geschichte seit Bismarck gewesen sei, und trug zur Einbindung der Bundesrepublik in die atlantische Gemeinschaft, vor allem durch ständigen wissenschaftlichen und politischen Austausch, bei (BHB II).

11 Hans Staudinger (1889-1980), Regierungsbeamter, Staatssekretär im Preußischen Handelsministerium; 1912 SPD, 1932-1933 MdR; 1933 in Haft, Emigration über Belgien, Frankreich und England in die USA; Mitglied der „German Labor Delegation“, Mitunterzeichner der Erklärung ehemaliger sozialdemokratischer Reichstagsabgeordneter von Januar 1947 gegen Massenvertreibung, Demontage und Besetzung (BHB I).

12 Norbert Muehlen (Muhlen) (1909-1981), Journalist und Schriftsteller, spielte eine herausragende Rolle bei der Organisation studentischer Proteste gegen den Nationalsozialismus, emigrierte 1933 in die Schweiz und 1934 ins Saargebiet, Redakteur der Exilzeitungen Westland und Grenzland; spielte ab 1935 in Paris neben Leopold Schwarzschild eine führende Rolle im antikommunistischen „Bund Freie Presse und Literatur“, war 1939-1940 interniert und gelangte 1941 in die USA (BHB II).

13 Alexander Böker (1912-1997) emigrierte 1938 in die USA, u. a. Gründer des „Committee against Mass Expulsions“; 1948 Rückkehr, Tätigkeit im Bizonen-Wirtschaftsrat und als Referent von Herbert Blankenhorn, später im diplomatischen Dienst, zuletzt Botschafter beim Vatikan (BHB I - Nachlaß im IfZ, ED 448). Weitere Emigranten im Council waren: Joseph Kaskell (BHB II), Gershon Canaan (BHB II), Felix R. Hirsch (BHB II), Hermann George Kaiser (BHB I), Stephen Kellen (BHB I), Carl Landauer (BHB II), Franz M. Oppenheimer (BHB II); Fritz E. Oppenheimer (BHB I), Henry M. Pachter (BHB II), Eric Waldmann (BHB II), Henry Wallich (BHB I) sowie der Politikwissenschaftler Carl J. Friedrich (1901-1984), der allerdings bereits in den zwanziger Jahren in die USA gekommen war und dort nach der NS-Machtübernahme blieb; Friedrich war nach 1945 Berater von OMGUS und 1956-1966 Professor an der Universität Heidelberg (Radkau, Deutsche Emigration, S. 250 und 274).

14 Alexander Böker an Christopher Emmet, 6. 8.1948 (IfZ, NL Marcia Kahn, Bd. 5); ebenso das Schreiben Karl Brandt an Emmet vom 21. 10. 1948, ebd. In einem Brief an Schauff geht Thieme auf das Thema „Umerzieher" ein „vom Schlage des Herrn ,Habe“, d.h. Hans Be- 
Dieser Einsatz für Westdeutschland und später die Bundesrepublik Deutschland stand in engem Kontext mit dem antikommunistischen Engagement des Councils, der die Westorientierung Bonns für unabdingbar hielt. Schon sehr früh nahm der Council auch die öffentliche Diskussion um eine Wiederbewaffnung der Bundesrepublik auf. Er vermittelte Kontakte zwischen amerikanischen und deutschen Politikern, Parlamentariern, Wirtschaftlern und Publizisten und organisierte deutsch-amerikanische Konferenzen, die alle zwei Jahre wechselnd in den USA und der Bundesrepublik stattfanden. An diesen Treffen beteiligten sich auf deutscher Seite der Bundeskanzler, die Außen- und Verteidigungsminister, die Präsidenten des Bundestages sowie weitere prominente Persönlichkeiten aus Politik, Wirtschaft, Wissenschaft und Publizistik, von amerikanischer Seite u.a. die Außenminister Dean Acheson und später Christian Herter und Henry Kissinger $^{15}$ sowie Persönlichkeiten wie General Clay, George Ball und James Conant ${ }^{16}$.

Dieser jahrelange Dialog hatte eine breite publizistische und öffentliche Resonanz, auf deutscher Seite war 1952 in Hamburg parallel die Organisation der "Atlantik-Brücke" gegründet worden. Auch diese deutsche Organisation, in der konservative Emigranten und Remigranten eine bedeutende Rolle spielten - die ersten beiden Vorsitzenden waren Ernst Friedländer und Arnold Bergstraesser ${ }^{17}$, weitere Mitglieder waren u. a. Max Brauer ${ }^{18}$ und Klaus Dohrn -, war bemüht, das gesellschaftliche und politische Image Deutschlands in den USA zu verbessern. Auf regelmäßigen deutsch-amerikanischen Konferenzen und Seminaren und mit einem breiten Publikationsprogramm, das sich unter anderem auch an die amerikanischen Soldaten in Deutschland wandte, wurde das vom American Council on Germany geknüpfte Netzwerk von Politikern, Vertretern der Wirtschaft, Wissenschaftlern und Journalisten noch vertieft und erweitert ${ }^{19}$.

kessy, ... deren Lebensberuf das Erziehen im der Hitlerei entgegengesetzten Sinn ist." (28. 7. 1945, IfZ, NL Schauff, Bd. 8).

15 Acheson war 1949-1953 amerikanischer Außenminister, Vertreter einer Politik des „containment " gegenüber der Sowjetunion; Herter war 1959-1961 Außenminister und Kissinger 1973-1977.

16 Conant war 1953-1955 amerikanischer Hoher Kommissar in der Bundesrepublik, 19551957 Botschafter; Ball war eine einflußreiche politische Persönlichkeit in den USA mit politischen Aktivitäten vor allem im Bereich der Europa- und Deutschlandpolitik.

17 Biographische Informationen zu Friedländer und Bergstraesser siehe unten, S. 212, Anm. 55 sowie S. 205, Anm. 13.

18 Max Brauer (1887-1973), hanseatischer Kommunalpolitiker (SPD), vor 1933 Oberbürgermeister von Altona, 1933 Emigration, ab 1936 in den USA; einer der Hauptvertreter der "rechten" sozialdemokratischen Emigration um die Neue Volkszeitung, die sich gegen die Kollektivschuld-These wandte, 1946 Rückkehr, ab 1946 Mitglied Hamburger Senat und 1946-1953 sowie 1957-1961 Erster Bürgermeister von Hamburg (BHB I).

19 An den Aktivitäten der „Atlantik-Brücke“ (Konferenzen etc.) waren von deutschen Emigranten/Remigranten die meisten beteiligt, die auch im Rahmen des „American Council on Germany" aktiv waren; darüber hinaus sind zu nennen: Wenzel Jaksch und Karl Mommer, außerdem Henry Kissinger, Alfred von Klemperer, Joachim Prinz, Hans Wallenberg, Arnold Wolfers (alle BHB I, Wolfers BHB II). Von den Persönlichkeiten in diesem politischen Netzwerk, die nicht emigriert waren, sind u. a. zu nennen: Kurt Birrenbach (allerdings 1939-1950 als Vertreter amerikanischer und deutscher Firmen in Buenos Aires/Argentinien), Erik Blumenfeld (stellvertretender Vorsitzender der „Atlantik-Brücke“), Heinrich von Brentano, Heinrich Deist, Marion Gräfin Dönhoff, Fritz Erler, Karl Theo- 
Die Vertreibung der Deutschen aus Osteuropa hatte der American Council on Germany in den USA schon früh angeprangert und ins öffentliche Bewußtsein gebracht ${ }^{20}$. Die ICMC sah nicht zuletzt in der Bewältigung der Konsequenzen dieses Exodus eine ihrer wesentlichen Aufgaben und war in dieser Frage über Johannes Schauff auch mit dem Council in Verbindung getreten ${ }^{21}$. Indessen weitete Schauff seine Tätigkeit für die ICMC über die reine Flüchtlingshilfe und Migrationsorganisation noch weiter aus. Durchaus im Einklang mit der konservativen Emigration zielten seine Bemühungen darauf ab, die Entwicklung Deutschlands bzw. der Bundesrepublik zu unterstützen und ihre politische Aufwertung und Einbindung in die westliche Welt zu befördern.

Auf mehreren internationalen kirchlichen Laienkongressen sowie im Zusammenhang mit dem von ihm mitvorbereiteten Eucharistischen Weltkongreß 1955 in Rio de Janeiro konnte er sich auf diplomatischer Ebene in die Vertretung der Interessen der Bundesrepublik einschalten. Bei solchen Gelegenheiten machten deutsche Politiker wie Kurt Georg Kiesinger und Helene Weber ihre ersten Gehversuche und Erfahrungen im Ausland nach dem Krieg. Bei seinen Bemühungen, Politiker der Bundesrepublik Deutschland in internationale Kommunikationszusammenhänge einzubinden, beklagte Schauff allerdings immer wieder deren aus der NS-Vergangenheit resultierende politische Verunsicherung: „Ich mußte häufiger den deutschen Standpunkt deutlicher vertreten, weil die deutschen Vertreter durch Leisetreterei und zu viele Konzessionen Erfolg zu haben glaubten. Ein politisches Selbstbewußtsein stellte sich bei vielen erst spät ein."22

Eine Gelegenheit, solchen Defiziten abzuhelfen, ergab sich auch, als Schauff 1961 durch Brentano in den von dem Außenminister installierten Kulturpolitischen Beirat des Auswärtigen Amtes berufen wurde 23 . In diesem Gremium, das von Dieter Sattler, damals Leiter der Kulturabteilung des Auswärtigen Amtes, geleitet wurde ${ }^{24}$, traf er auf alte Bekannte und politische Freunde wie Maria Schlüter-Hermkes, Theodor Heuss, Josef Pieper und Arnold Bergstraesser.

dor von und zu Guttenberg, Richard Jaeger, Kurt Georg Kiesinger, Hermann Kopf, Carl Otto Lenz, Kurt Mattick, Erich Mende, Klaus Mehnert, Hans Möller, Gerd Ruge, Heinz Ruhnau, Karl Schiller, Carlo Schmid, Helmut Schmidt, Hermann Schmitt-Vockenhausen, General Johannes Steinhoff, Otto Wolff von Amerongen. Namenslisten in: Fünfzehn Jahre Atlantik-Brücke (American Council on Germany). Hamburg 1967.

$20 \mathrm{Vgl}$. auch die entsprechenden Aktivitäten von Alexander Böker im „Committee against Mass Expulsions" (siehe Anm. 13).

21 Mittelsmann war hier vor allem Klaus Dohrn; vgl. die Korrespondenz in IfZ, NL Schauff, Bd. 2 u. 32.

22 Interwiew Erich Kusch mit Johannes Schauff, November 1974 (Entwurf mit handschriftlichen Ergänzungen Schauffs; eine gekürzte Fassung wurde später im Südwestfunk gesendet; Ms. im Besitz des Verfassers).

23 Schreiben Heinrich von Brentano an Schauff vom 21. 11.1960, in dem der Außenminister die Gründung eines solchen Gremiums ankündigt, in das 15 bis 20 Persönlichkeiten berufen werden sollen und das vor allem die Kulturarbeit im Ausland (Schulen, Sprache etc.) erörteren soll (IfZ, NL Schauff, Bd. 1). Schauff gehörte dem Kulturpolitischen Beirat bis 1973 an. Schreiben des damals amtierenden Außenministers Walter Scheel an Johannes Schauff vom 31. 8. 1973 (IfZ, NL Schauff, Bd. 37).

24 Dieter Sattler (1906-1968), ursprünglich Architekt, war ab 1947 Staatssekretär im Bayerischen Kultusministerium, in dieser Eigenschaft an der Gründung des Instituts für Zeitge- 
Politische Zielsetzung dieses Kulturpolitischen Beirats war eine „Bildungshilfe als Grundlage der Entwicklungshilfe" für die Dritte Welt. Bei der Planung einer Offensive vor allem auf dem Schul- und allgemeinen Bildungssektor, die auch einer Verbesserung und Differenzierung des Deutschlandbildes im Ausland dienen sollte, wies Schauff auf die „erwachende Landarbeiterschaft Lateinamerikas“ hin, die von Europa Leit- und Ordnungsbilder erwarte in Hinblick darauf, daß Moskau dieses Feld zunehmend besetze; Castro sei da ein warnendes Beispiel25. Da den USA das Landarbeiterproblem, wie es sich in Lateinamerika stelle, fremd sei, habe die Entwicklungshilfe der europäischen Länder hier eine große Aufgabe.

Schauff engagierte sich in diesem Zusammenhang für eine kulturpolitische $\mathrm{Ge}$ genoffensive zu der kommunistischen Einflußnahme vor allem in Lateinamerika, dessen Verhältnisse ihm ja vertraut waren. Zugleich warnte er jedoch vor der Tatsache, daß ein nicht unbeträchtlicher Teil der deutschen Auslandslehrer in Lateinamerika nach wie vor aus ehemaligen Nationalsozialisten bestehe ${ }^{26}$. Seine Gegnerschaft zu totalitären Systemen war eben nicht teilbar. Als Repräsentanten eines besseren Deutschland empfahl er bei der vorgesehenen kulturpolitischen Offensive deutschsprachige Emigranten sowie Vertreter der Migration aus der Nachkriegszeit, für die das Deutsche die "europäische Sprache" sei27.

Schauffs Status bei dieser Mitarbeit im Kulturpolitischen Beirat des Auswärtigen Amtes wurde schließlich auch nach außen hin weiter aufgewertet, als ihm im Zuge seines Wiedergutmachungsverfahrens 1959 der Rang eines Ministerialdirigenten zuerkannt wurde. Zum positiven Ausgang dieses Verfahrens hatte wesentlich Schauffs Schwager, der Freiburger CDU-Abgeordnete Hermann Kopf, beigetragen ${ }^{28}$ : Schauffs Berufsweg und den Tatbestand der Verfolgung durch das NS-Regime bezeugten weitere alte politische Weggefährten wie Heinrich Krone, Josef Franken, Edmund Forschbach, Wilhelm Boyens und Karl Fütterer. Die Anerkennung des Ministerialdirigenten-Rangs beim Bundesministerium für Ernährung, Landwirtschaft und Forsten erfolgte auf der Grundlage von Schauffs Laufbahn in der GFK vor 1933, die Hochrechnung der Dienstbezüge erbrachte am 26. Juni 1959 die Beförderung zum Ministerialdirigenten (Besoldungsgruppe B 7 a $\mathrm{RBO}$ ) rückwirkend zum 1. April 1950. Schauff optierte für eine rückwirkende Pensionierung und führte den Titel eines Ministerialdirigenten a.D., dem für weitere Unternehmungen ein Ministerialpaß zur Verfügung gestellt wurde29.

schichte beteiligt; 1952 Kulturreferent der deutschen Botschaft in Rom, ab März 1959 als Ministerialdirektor mit der Leitung der Kulturabteilung des AA betraut. Sattler war vor 1933 eng mit der kath. Jugendbewegung verbunden. Sein Nachlaß befindet sich im Institut für Zeitgeschichte (IfZ, ED 145).

25 Zur kommunistischen Bedrohung in Lateinamerika vgl. auch die Korrespondenz SchauffSattler in IfZ, NL Schauff, Bd. 7 sowie NL Sattler, Bd. 90.

26 Protokoll der 1. Sitzung des Kulturpolitischen Beirats (12. und 13. Januar 1961), PAAA 600/IV 1, Bd. 299; vgl. auch den Kommentar von Friedrich Sieburg in der FAZ vom 23. 1. 1961.

27 Protokoll der 2. Sitzung des Kulturpolitischen Ausschusses (1. und 2. Mai 1961), PAAA $600 /$ IV 1, Bd. 299, S. 10 f.

$28 \mathrm{Vgl}$. den betreffenden Briefwechsel zwischen Kopf, Schauff u.a. in: ACDP, NL Hermann Kopf, Sign. I-027-004/3.

29 Der Wiedergutmachungsvorgang in IfZ, NL Schauff, Bd. 27; vgl. auch die Korrespondenz 
Schauffs Tätigkeit für die GFK, einer Gesellschaft des bürgerlichen Rechts und später ein eingetragener Verein, dem öffentlichen Dienst zuzurechnen, war eine ausgesprochene Kulanz der mit dem Wiedergutmachungsverfahren befaßten Stellen. Seine frühere Tätigkeit für das Statistische Reichsamt und auch seine spätere vorübergehende Berufung in das Innenministerium in Zusammenhang mit der Ausarbeitung eines Konzepts für eine Wahlrechtsreform ${ }^{30}$ dienten allerdings in diesem Zusammenhang als gewichtige zusätzliche Argumente, so daß die Wiedergutmachungsbehörden anerkennen konnten, der Antragsteller sei „als Angehöriger des öffentlichen Dienstes aus Gründen politischer Gegnerschaft gegen den Nationalsozialismus durch nationalsozialistische Gewaltmaßnahmen verfolgt und dadurch in seinem Arbeitsverhältnis geschädigt worden“. Die Entwicklung seiner Laufbahn nach 1933 hätte - so die Annahme der Wiedergutmachungsbehörde - dazu geführt, „daß er ohne Schädigung aller Voraussicht nach bei der Auflösung der Reichsstelle für Siedlerberatung im Herbst 1933 in das Reichsministerium für Ernährung und Landwirtschaft" übernommen worden wäre. Auch daß der Antragsteller sein Studium „nur" mit dem Dr. phil. abgeschlossen und keine Staatsprüfung abgelegt habe, wurde nicht als Hindernis für eine "Anstellung in der Laufbahn des höheren Dienstes“ gewertet. Somit werde als „Zeitpunkt der Ernennung zum Regierungsrat ... der 1. Oktober 1934 festgesetzt“. Dies ergab bei Hochrechung normaler Beförderungsstufen das Erreichen einer Ministerialdirigentenstelle im Jahr 1950.

\section{Internationale christdemokratische Politik}

Do ut des! So wie die Bundesrepublik bei ihrem Weg in die Demokratie westlichen Zuschnitts Unterstützung fand, war die Regierung nach einer ersten Konsolidierungsphase ihrerseits bemüht, Staaten politische und wirtschaftliche Entwicklungshilfe zukommen zu lassen, die es - vor allem in Lateinamerika - gegen totalitäre Anfechtungen zu immunisieren galt. Die Organisation einer solchen Entwicklungshilfe war jedoch zugleich auch ein Schritt hin zur europäischen Zusammenarbeit und zur internationalen Anerkennung und Aufwertung der Bundesrepublik. In diesem Zusammenhang konnte Schauff aufgrund seiner lateinamerikanischen Erfahrungen und Verbindungen eine politische Schlüsselstellung einnehmen.

Am 14. Juni 1963 wurde Schauff in den Vorstand des „Instituts für internationale Solidarität" (IIS) berufen. Das IIS war ein Ableger der Politischen Akademie Eichholz, einer Stiftung der CDU, die 1957 gegründet worden war und später in

Schauff-Krone sowie die Korrespondenz mit Kiesinger (vor allem Schreiben Schauffs vom 11. 2. 1967) betr. Verlängerung des Ministerialpasses in Anbetracht der Wahrnehmung von konkreten und informellen "Verbindungsaufgaben im deutschen Regierungsinteresse“ vor allem im Ausland (IfZ, NL Schauff, Bd. 4).

30 Siehe oben, S. 48. 
der Konrad-Adenauer-Stiftung aufging ${ }^{31}$. Ähnlich wie die SPD und später auch andere Parteien versuchte die CDU auf diese Weise ihre politische Bildungsarbeit $\mathrm{zu}$ institutionalisieren und damit zu professionalisieren. Die Aufgabe dieser Institutionen bestand darüber hinaus in der Schulung von Funktionsträgern und sogenannter gesellschaftlicher Multiplikatoren.

Eine zentrale Rolle bei der Institutionalisierung dieser politischen Außenarbeit spielten der damalige Bundesgeschäftsführer der CDU, Bruno Heck, sowie der Vorsitzende des Trägervereins, Professor Arnold Bergstraesser. Vor allem Bergstraesser kommt das Verdienst zu, daß sich die Tätigkeit der Akademie nicht in interner Parteischulung erschöpfte, sondern auch nach außen gesellschaftspolitisch initiativ wurde. Dies schloß angesichts der europäischen und internationalen politischen Entwicklung nahezu zwingend auch internationale Perspektiven ein.

Die internationale Situation war zu Beginn der sechziger Jahre vor allem durch das Entstehen und rapide Anwachsen der Befreiungsbewegungen in der Dritten Welt, den Amtsantritt der Kennedy-Regierung in den USA (1960) und die Revolution Fidel Castros in Kuba (1961) geprägt. Waren für die CDU im Verhältnis zur Dritten Welt bisher vor allem handelspolitische Interessen maßgeblich gewesen, so daß sie auf dem politischen Terrain der SPD bzw. der Friedrich-Ebert-Stiftung mit ihren Verbindungen zur Sozialistischen Internationale und dem Internationalen Bund Freier Gewerkschaften (IBFG) kaum Konkurrenz gemacht hatte, so wurde Ende der fünfziger Jahre die Hilfe für die Entwicklungsländer zentraler Bestandteil christdemokratischer Politik ${ }^{32}$. Dies wurde sicherlich auch durch die Forderung der neuen Kennedy-Administration nach Unterstützung insbesondere der lateinamerikanischen Länder befördert, die zunehmend von kommunistischen Umsturzversuchen bedroht schienen. Ein weiteres Motiv für eine nationale Entwicklungspolitik war die Beteiligung der Bundesrepublik an dem Europäischen Entwicklungsfonds, der im Zuge der Gründung der Europäischen Wirtschaftsgemeinschaft eingerichtet wurde und bei dem zunächst der französische Einfluß vorherrschte, den es auch im bundesdeutschen Interesse zu relativieren galt.

Nach den Bundestagswahlen 1961 wurde schließlich für diesen Bereich des Regierungshandelns ein eigenes Ministerium, das Bundesministerium für wirtschaftliche Zusammenarbeit (BWZ), eingerichtet, dem bis 1966 Walter Scheel als Minister vorstand und das damit bis 1966 zu einer Domäne des Koalitionspartners FDP wurde. Schauff war mit dieser Entwicklung keineswegs einverstanden; er drängte daher auf einen entsprechenden Kompetenzzuwachs der CDU-Stiftung ${ }^{33}$. Im September 1961 nahm Peter Molt als Vertreter der Stiftung innerhalb der deutschen Delegation in Luzern am 15. Kongreß der „Nouvelles Equipes Internationales" teil, auf die weiter unten noch einzugehen sein wird. Dort konnten erste Kontakte mit lateinamerikanischen Christdemokraten aufgenommen wer-

31 IfZ, NL Schauff, Bd. 22. Hier besonders das Manuskript von Peter Molt „Die ersten Jahre der Konrad-Adenauer-Stiftung “, o. D.

32 Vgl. Scholz, Entwicklungspolitik.

33 Schreiben Schauff an das Generalsekretariat der CDU (Peter Molt) vom 13. 11. 1961. IfZ, NL Schauff, Bd. 35. 
den. Eine besondere Rolle bei der Ausformulierung einer eigenen christdemokratischen Entwicklungspolitik spielte der Belgier August Vanistendael, zu diesem Zeitpunkt Generalsekretär des Internationalen Christlichen Gewerkschaftsbundes (IFCTU) und Mitarbeiter Schauffs in der ICMC, der viel dazu beitrug, daß sich schließlich auch Bundeskanzler Adenauer entwicklungspolitisch engagierte $^{34}$.

August Vanistendael hatte als einer der ersten ausländischen Politiker Kontakt mit der CDU gesucht und war mit Adenauer bereits während des Recklinghauser Parteitages der CDU der britischen Zone 1948 zusammengetroffen. Er hatte zu den Befürwortern einer Neugründung der christlichen Gewerkschaften in der Bundesrepublik gehört und bereits in den fünfziger Jahren die These vertreten, daß sich die christlichen Gewerkschaften vor allem in Lateinamerika und Afrika als ein Gegengewicht gegen den Kommunismus etablieren müßten. Die Gründung der Konföderation Christlicher Gewerkschaften 1954 in Chile geriet allerdings in Gegensatz zur amerikanischen Gewerkschaft $\mathrm{AFL} / \mathrm{CIO}^{35}$ und dem IBFG mit ihrer prononciert antiklerikal-laizistischen Haltung, die wiederum von der Friedrich-Ebert-Stiftung - mit Zuwendungen des Auswärtigen Amtes unterstützt wurde. Obwohl Adenauer den Erfolg neugegründeter christlicher Parteien und Gewerkschaften in Lateinamerika skeptisch beurteilte, vermittelte er Vanistendael damals für deren Unterstützung erhebliche Mittel ${ }^{36}$. Ende November 1961 fand in der Politischen Akademie Eichholz eine Fachtagung über das geplante entwicklungspolitische Engagement statt, deren Ergebnis der Grundsatz einer partnerschaftlichen Zusammenarbeit mit weltanschaulich "verwandten" Parteien und Gewerkschaften in der Dritten Welt war. Zur Durchführung dieses Konzepts wurde unter dem Dach des Vereins Politische Akademie Eichholz ein „Institut für Internationale Solidarität" konzipiert.

Auf Initiative Vanistendaels, der im Januar 1962 nach Venezuela gereist war und anschließend ein Memorandum an Adenauer verfaßt hatte, in dem er auf die Bedrohung Lateinamerikas durch die kubanische Revolution und die deshalb wachsende Bedeutung der christlich-demokratischen Parteien hinwies ${ }^{37}$, stattete Rafael Caldera, damals Präsident des venezolanischen Parlaments, am 8. Februar 1962 Konrad Adenauer einen Besuch ab, der als ein erstes Hilfsprojekt die Unterstützung des gewerkschaftlichen Bildungsinstituts INES ${ }^{38}$ in Caracas zusagte. Im Gegenzug erfolgte durch Caldera eine Einladung für den im Mai 1962 stattfinden-

34 Vgl. dazu Adenauers Gespräche mit David Ben Gurion am 14.3.1960 sowie mit General de Gaulle am 23.1. 1963; bei dieser Gelegenheit schlug Adenauer vor, eine gemeinsame Politik gegenüber Lateinamerika zu entwickeln, um den Subkontinent vor dem Kommunismus zu retten (Adenauer, Erinnerungen, S. $37 \mathrm{ff}$. und S. 210; Molt, Die ersten Jahre ..., Ms., IfZ, NL Schauff, Bd. 22, S. $17 \mathrm{ff}$.).

35 AFL/CIO - American Federation of Labor/Congress of Industrial Organizations.

36 Gespräch Peter Molt mit Vanistendael am 15. 5. 1988 in Leuven, zit. in Molt, Die ersten Jahre ..., Ms., IfZ, NL Schauff, Bd. 22, S. 19.

37 Ebenda, S. 21 f.; das Memorandum in: Katholische Universität Leuven, NL August Vanistendael.

38 INES - Instituto Nacional de Estudios Sindicales (Institut für nationale Gewerkschaftsstudien, den christlichen venezolanischen Gewerkschaften angeschlossen). 
den 1. Weltkongreß der Internationalen Union der Jungen Christlichen Demokraten, zu der auch eine CDU-Parteidelegation erschien ${ }^{39}$.

Nach diesem ersten Schritt wurde das Projekt einer lateinamerikanischen Entwicklungshilfe auch auf bundespolitischer Ebene befördert. Das Bundeskanzleramt, das Auswärtige Amt und das Bundesministerium für wirtschaftliche Zusammenarbeit (BWZ) vereinbarten zwei Haushaltstitel für die internationale Arbeit der Akademie Eichholz, die aber auch der Friedrich-Ebert-Stiftung und der Friedrich-Naumann-Stiftung zukommen sollten - ein Titel für "gesellschaftspolitische Maßnahmen“ in den Entwicklungsländern lag beim BWZ, der andere zur „Förderung des demokratischen Gedankens im Ausland“ beim Außenministerium.

Damit war der Weg zur Gründung des „Instituts für Internationale Solidarität“ (IIS) im organisatorischen Verbund mit der Akademie Eichholz geebnet. Zu seiner Leitung wurde ein Institutsvorstand berufen, der sich in einen geschäftsführenden und einen erweiterten Vorstand gliederte. Das Projekt wurde schließlich von Adenauer gebilligt, nachdem seine Bedenken, der Begriff „Solidarität“ klinge zu „sozialistisch“, ausgeräumt werden konnten ${ }^{40}$. Am 1. Juli 1962 fand die konstituierende Sitzung des neuen Instituts statt. Dem ersten Vorstand gehörten die Bundestagsabgeordneten Gerhard Fritz, Heinrich Gewandt, Bruno Heck, Walter Althammer und Gerhard Stoltenberg sowie der ehedem ebenfalls in Brasilien exilierte Hermann Mathias Goergen an; ein Jahr später erfolgte dann die Berufung von Johannes Schauff ${ }^{41}$.

Lateinamerika wurde zum Arbeitsschwerpunkt des IIS. Dies hatte eine Reihe von politischen und organisatorischen Gründen, hing aber auch mit der zentralen Rolle zusammen, die Schauff und Goergen als Brasilienemigranten spielten. Während in Afrika und Asien die Partnersuche vor allem aufgrund der fortbestehenden Bindungen der neuen Staaten an die ehemaligen Kolonialmächte schwierig war, gab es in Lateinamerika christlich-demokratische Parteien und Gewerkschaften, die zur Christlich-Demokratischen Weltunion und zum Weltverband der christlichen Gewerkschaften gehörten. Mit diesen Partnern war trotz manchmal unterschiedlicher politischer Zielsetzung ein Dialog auf der Grundlage von Subsidiarität und Solidarität möglich. Die Hilfe für diese Parteien war überdies eingebunden in die Zusammenarbeit mit den "Nouvelles Equipes Internationales" (NEI) ${ }^{42}$ : Damit konnte dem häufig geäußerten Verdacht, die Tätigkeit des

$39 \mathrm{Zu}$ der Delegation gehörten Peter Molt und der Bundestagsabgeordnete Heinrich Gewandt sowie Vanistendael und der Leiter des Internationalen Christlichen Studien- und Dokumentationszentrums in Rom, Karl Josef Hahn. Molt, Die ersten Jahre ..., Ms., IfZ, NL Schauff, Bd. 22, S. 23 f., sowie Bd. 23.

40 Der geschäftsführende Vorsitzende des Vereins Akademie Eichholz, Kraske, konnte den Kanzler davon überzeugen, daß der Begriff auch in der katholischen Soziallehre durchaus gängig war, wie z. B. bei dem Jesuiten Heinrich Pesch („Solidarismus“). Molt, Die ersten Jahre ..., Ms., IfZ, NL Schauff, Bd. 22, S. 24.

41 Vgl. das Berufungsschreiben von Staatssekretär a. D. Thedieck vom 2. 8.1965 (IfZ, NL Schauff, Bd. 22).

42 „Nouvelles Equipes Internationales“: 1947 erfolgter Zusammenschluß der christlichdemokratischen Parteien Westeuropas, erster Kongreß und Namensgebung 1947 in Clau- 
IIS folge vorrangig nationalstaatlichem deutschem Interesse, wirksam begegnet werden ${ }^{43}$.

Der Schwerpunkt Lateinamerika war zunächst jedoch nicht unumstritten. Schauff und Goergen, die beide häufig vor Ort die neueste Entwicklung studieren konnten, vermochten jedoch Konrad Adenauer entsprechend zu beeinflussen, so $\mathrm{da}$ dieser bis zum Ende seiner Amtszeit als Kanzler seine ganze Autorität für das Projekt "Lateinamerika“ in die Waagschale warf. Auch Bruno Heck und das spanischsprachige Mitglied des Haushaltsauschusses, Heinrich Gewandt, spielten eine bedeutende Rolle. Adenauer persönlich traf die Entscheidung, den Wahlkampf des christlich-demokratischen Präsidentschaftskandidaten in Chile, Eduardo Frei, mit einer erheblichen Geldsumme zu unterstützen ${ }^{44}$. Dabei ging es nicht nur um einen Beitrag zur politisch-sozialen Schulung und finanzielle Unterstützung; über das IIS wurde auch ein intensiver Austausch von deutschen und lateinamerikanischen Politikern ermöglicht. Auf deutscher Seite waren daran vor allem damals noch junge Politiker wie Egon Klepsch, Bernhard Vogel, Heiner Geißler und Jürgen Wohlrabe beteiligt, auf lateinamerikanischer Seite Rafael Caldera und Eduardo Frei ${ }^{45}$.

Die Aktivitäten des IIS berührten natürlich auch die „Nouvelles Equipes Internationales“, die 1961 in der „Union Mondiale Chrétienne“ aufgingen ${ }^{46}$. Von 1963 bis 1966 war auch das IIS in diesem Gremium vertreten. Die Notwendigkeit, die Zusammenarbeit der christdemokratischen Parteien zu fördern und ihre Arbeit zu koordinieren, betraf in erster Linie Lateinamerika, wo traditionelle Bindungen der italienischen Democrazia Cristiana (DC) bestanden. Deren Vertreter Sereno Freato und Angelo Bernassola nahmen 1963 Kontakte mit Peter Molt und August Vanistendael auf. Eine wichtige Rolle spielte dabei auch das „Centre International Démocrate-Chrétien d'Etudes et de Documentation“ in Rom, das von Karl Josef Hahn geleitet wurde.

Am 31. März 1965 wurde in Rom die „Fondation Internationale de Solidarité “ (FIS) gegründet - Gründungsmitglieder waren Molt, Freato und Vanistendael, den Vorsitz übernahm auf persönliches Drängen Adenauers Johannes Schauff47. Die FIS wurde im wesentlichen von den christlich-demokratischen Parteien Italiens, Deutschlands, der Niederlande und später auch Venezuelas und Chiles ge-

defontaine bei Lüttich/Belgien. Vgl. Karl Josef Hahn, Die internationale Zusammenarbeit der christlich-demokratischen Parteien in Europa, Ms. (1964), IfZ, NL Schauff, Bd. 23.

43 Vgl. Lothar Kraft, Partner der gesellschaftspolitischen Zusammenarbeit, in: Silke Krieger (Hrsg.), Partner, S. 14; Molt, Die ersten Jahre ..., Ms., IfZ, NL Schauff, Bd. 22, S. 25 f.

44 Schauff erinnert sich, daß seinerzeit Staatssekretär Westrick einen Scheck in Höhe von DM 200000 überreicht habe (Notiz vom 28. 8. 1968, IfZ, NL Schauff, Bd. 39); Molt weist darauf hin, daß diese Spende über das IIS geleitet wurde und nicht, wie seinerzeit im Spiegel behauptet (Nr. 38/1964), über die Misereor-Kollekte (Molt, Die ersten Jahre ..., Ms., IfZ, NL Schauff, Bd. 22, S. 28).

45 Caldera wurde 1965 zum CDU-Parteitag in Düsseldorf eingeladen und sprach dort als erster offizieller lateinamerikanischer Gast ein Grußwort. Vom 17. bis 22.7. 1965 erfolgte der Staatsbesuch des neugewählten Präsidenten Chiles Eduardo Frei, der auch der Vorstellung lateinamerikanischer christlich-demokratischer Politik diente.

$46 \mathrm{Vgl}$. Portelli/Jansen, Démocratie chrétienne, S. 31-54.

47 IfZ, NL Schauff, Bd. 23 u. 39 (Gründungsdokumente und Berufung Schauffs). 
tragen. Schauff war bemüht, auch die Christdemokraten aus Österreich, Belgien, Frankreich und der Schweiz einzubinden. 1965 wurde ein Exekutiv-Komitee gebildet, dem die Gründungsmitglieder, der Vorsitzende und ein Exekutiv-Sekretär - zunächst der Pole Stanislaw Gerhardt - angehörten. Am 4. September 1965 fand die erste Sitzung der allgemeinen Mitgliederversammlung statt, auf der auch die Vertreter Österreichs, Belgiens und der Schweiz teilnahmen. Schauff wurde offiziell zum Vorsitzenden der Fédération gewählt. Insgesamt wurden 21 Sitzungen des Exekutivkomitees einberufen sowie drei allgemeine Mitgliederversammlungen in Taormina, Rom und Venedig. Die Zusammensetzung der Spitze änderte sich später: an die Stelle von Sereno Freato trat Angelo Bernassola und an die von Peter Molt Adolf Herkenrath, der die Leitung des IIS übernommen hatte ${ }^{48}$; das IIS wurde zugleich Mitglied der FIS. 1968 zog sich Vanistendael aus dem Exekutiv-Komitee zurück, und die österreichischen, belgischen und Schweizer Parteien verließen die Fédération aufgrund eigener finanzieller Schwierigkeiten. An ihre Stelle traten die Niederlande, Chile und Venezuela.

Hauptziel der FIS war die Koordination der Zusammenarbeit mit den jungen, im Aufbau befindlichen christlich-demokratischen Bewegungen und Institutionen vor allem in Lateinamerika; hierfür sollte die Unterstützung durch Kreise außerhalb der christlich-demokratischen Parteien gewonnen werden ${ }^{49}$. Konkret geplant und vorbereitet wurden Maßnahmen zur politischen und gewerkschaftlichen Schulung vor Ort und im Austauschverfahren, internationale Konferenzen sowie Untersuchungen und Studien über soziale, kulturelle, politische und wirtschaftliche Fragen. In Lateinamerika konnte die FIS mit verschiedenen bereits existierenden staatsbürgerlichen Ausbildungszentren kooperieren, wobei zu Beginn die Zusammenarbeit mit dem IIS und der italienischen DC besonders fruchtbar war.

Innerhalb des IIS und der Fédération wandte Schauff sich aufgrund seiner gründlichen Kenntnis der Problemlage vor allem gegen Tendenzen, die Entwicklung in Lateinamerika allein unter einem sozialrevolutionären Blickwinkel zu beurteilen, wie sie sich in der europäischen Öffentlichkeit z.B. bei der Beurteilung des Sturzes der brasilianischen Regierung Goulart (März 1964) als gängige Meinung erwiesen hatten ${ }^{50}$. Im Februar 1965 wies er in diesem Zusammenhang in einem Gespräch mit Mariano Rumor, dem damaligen politischen Sekretär der DC, auch auf die Bedeutung einer aktiven Ostpolitik hin, die Möglichkeiten einer

48 Herkenrath war katholischer Jugendfunktionär, ab 1964 Bürgermeister der Stadt Siegburg.

49 Johannes Schauff, Bericht über die Tätigkeit der FIS in den Jahren 1963 bis 1971 vom Vorsitzenden Dr. Johannes Schauff, 24. 5. 1971 (IfZ, NL Schauff, Bd. 23).

50 Der brasilianische Präsident João Goulart war im März 1964 wegen seiner Pläne für eine Sozial- und Bodenreform durch einen Militärputsch gestürzt worden. Vgl. Schauffs Korrespondenz mit dem damaligen Botschafter der Bundesrepublik in Rio de Janeiro, Gebhard Seelos, 1964/65. Anläßlich einer Berichterstattung im Lateinamerikanischen Bulletin (Nr. 3, 30. 6. 1964) zu den Vorgängen, die sich den Standpunkt eines „reaktionären Staatsstreichs" der Christlichen Gewerkschaften zu eigen machte, warnte Schauff vor Neigungen der Christdemokraten zur Linken. „Ich habe bereits mit Minister Krone gesprochen, daß man diese Tendenzen als [eine] Krise der CDU nicht durchgehen lassen darf." (Schreiben vom 2. 9. 1964, IfZ, NL Schauff, Bd. 7). 
Einflußnahme von christlich-demokratischer Seite biete ${ }^{51}$. Nach Gründung der FIS wurde dann auch neben dem Engagement für Lateinamerika eine Aktion zur Stützung christlicher Demokraten hinter dem Eisernen Vorhang eingeleitet, die durch Übersetzung westlicher Literatur ins Polnische, Tschechische und Ungarische vor allem auf politische Information abzielte ${ }^{52}$. Eine analoge Aktion der FIS galt der Unterstützung und Stärkung der spanischen christlich-demokratischen Kräfte unter dem Franco-Regime ${ }^{53}$. Bei all diesen Aktionen war die Fédération gefordert, auch die nötigen Geldmittel zu beschaffen, die sich am Ende auf ca. 10 Millionen Dollar beliefen ${ }^{54}$.

Die Tätigkeit der FIS hing, wie schon angeführt, eng mit der Christlich-Demokratischen Weltunion (Union Mondiale Démocrate Chrétienne/UMDC) zusammen. Als diese auf ihrem 5. Weltkongreß am 23./24. April 1966 in Lima in eine Krise geriet, hatte dies auch unmittelbar Folgen für die FIS. Bei der Auseinandersetzung in Lima ging es vor allem um die politische und wirtschaftliche Bevormundung durch die USA und die Europäer, aber auch um die grundsätzliche Orientierung der christlichen Demokraten Lateinamerikas angesichts der nach der kubanischen Revolution sich verstärkt entwickelnden Guerillabewegungen und dem Entstehen der sogenannten Befreiungstheologie ${ }^{55}$. Diese Auseinandersetzung lähmte die Arbeit der UMDC zunächst nachhaltig; zu Beginn der siebziger Jahre konnte die Stagnation jedoch angesichts der besorgniserregenden Entwicklung in Chile und Uruguay, die 1973 schließlich zu Staatsstreichen von seiten des Militärs führen sollte, wieder überwunden werden. Die FIS, die einen entscheidenden Beitrag zu dieser Konsolidierung geleistet hatte, beendete danach ihre Tätigkeit ${ }^{56}$. „Ich glaube“, so vermerkte Schauff in seiner abschließenden Wertung der von ihm geleiteten Arbeit der FIS, „daß wir ein solides Fundament für eine zukünftige christlich-demokratische Zusammenarbeit ... geschaffen haben."

Schauffs Eintreten für eine christlich-demokratische Reformpolitik angesichts der sich ausbreitenden kommunistisch-diktatorischen Entwicklung wurde im damaligen Meinungsklima von einem Teil der Presse in der Bundesrepublik, die

51 Gedächtnisprotokoll der Besprechung Schauffs mit Rumor, an der auch Karl Josef Hahn teilnahm, vom 22. 2. 1965 am Sitz der DC, Piazza Luigi Sturzo (IfZ, NL Schauff, Bd. 23).

52 Schauff war darum bemüht, über Staatssekretär Globke und Heinrich Krone auch die Amerikaner in diese Aktionen finanziell einzubinden. Vgl. den Brief Schauffs an Heinrich Krone vom 2. 8. 1965, IfZ, NL Schauff, Bd. 23.

$53 \mathrm{Vgl}$. Bericht Karl Josef Hahn an Schauff über eine Informationsreise durch Spanien, 18. 6. 1965 (IfZ, NL Schauff, Bd. 23); ebenso Schauffs „Notiz über die Zweckmäßigkeit einer Begegnung von Bundesminister Leber mit dem italienischen Verteidigungsminister Arnaldo Forlani“ vom 20. 9. 1970, in der auf die Rolle von Joaquín Ruiz-Giménez hingewiesen wird und die Notwendigkeit, eine Entwicklung wie in Portugal zu vermeiden (IfZ, NL Schauff, Bd. 39).

54 Schauff, Bericht über die Tätigkeit der FIS (1971), IfZ, NL Schauff, Bd. 23, S. 4.

55 Zur „Befreiungstheologie“ siehe, S. 171.

56 „Avec le FIS disparut une tentative d'aide multilatérale des PDC du Tiers Monde, et l'on favorisa de plus en plus une politique de solidarité qui tendait à devenir bilatérale “ (Brian Palmer, De l'Union Mondiale Démocrate Chrétienne à l'Internationale Démocrate-Chrétienne, in: Portelli/Jansen, Démocratie chrétienne, S. 41-54, hier: S. 47); Molt, Die ersten Jahre ..., Ms., IfZ, NL Schauff, Bd. 22, S. 50.

57 Schauff, Bericht über die Tätigkeit der FIS, IfZ, NL Schauff, Bd. 23, S. 4. 
von einer quasi unpolitischen Entwicklungspolitik ausging ${ }^{58}$, nie richtig - oder bewußt falsch - verstanden. Natürlich ist nicht zu bestreiten, daß in diesem Zusammenhang nicht nur die Unterstützung demokratischer Entwicklungen in Lateinamerika, sondern auch wirtschaftspolitische und wirtschaftsstrukturelle Gesichtspunkte eine Rolle spielten. Die Kritik an Politikern und Mitarbeitern des IIS wie Herkenrath, Moser ${ }^{59}$, Gewandt und Schauff, sie seien in „undurchsichtige Aktivitäten" verwickelt und würden Steuergelder zugunsten ihrer südamerikanischen politischen Klientel abzweigen, ja seien selbst Entwicklungshilfegewinnler, ist jedoch in keiner Weise haltbar. Analoge Vorwürfe wären auch gegenüber den Parteistiftungen von SPD und FDP möglich gewesen. Schauff, dem die „Hauptrolle" in dieser christdemokratischen Lateinamerika-Politik zugeschrieben wurde, konnte selbstverständlich aufgrund seiner Verbindungen als ehemaliger Emigrant und als politisch unabhängiger Mann wirtschaftliche Verbindungen knüpfen - so etwa im Zusammenhang mit der Entwicklung der venezolanischen Erdölindustrie oder der Etablierung von Verkehrsverbindungen der Deutschen Lufthansa ${ }^{60}$. Er leistete damit Aufbauarbeit im besten entwicklungspolitischen Sinne und trug zugleich zur Verbesserung deutscher außenwirtschaftlicher Beziehungen bei. Der Vorwurf, er habe zugleich im eigenen wirtschaftlichen Interesse gehandelt, ist bei Kenntnis der Persönlichkeit von Johannes Schauff schlichtweg abwegig. Hämische Anmerkungen in der Presse anläßlich hoher Auszeichnungen Schauffs in Venezuela und Brasilien ${ }^{61}$ galten nicht zuletzt seiner dezidiert christlich-demokratischen und antikommunistischen Grundhaltung. Diese zeigte sich auch in seiner klaren Ablehnung der lateinamerikanischen Befreiungstheologie.

58 Vgl. z. B. Der Spiegel 12/1971 („Bolivars Hilfe“, S. 37) und 12/1973 („Schmutzige Wäsche ist deren Sache“, S. 27-34). In diesen Artikeln wird vor allem die Rolle Schauffs hervorgehoben.

59 Gemeint ist Edmund Moser, der zusammen mit seinem Bruder Gerold Moser v.a. in Venezuela/Caracas tätig war. Aus beider Briefwechsel mit Schauff geht hervor, daß Schauff auch in den siebziger und achtziger Jahren weiterhin aktiven Anteil an der lateinamerikanischen politischen Entwicklung nahm (IfZ, NL Schauff, Bd. 5).

60 Vgl. Schreiben Schauff an den damaligen Chef des Bundeskanzleramts, Minister Horst Ehmke, vom 22. 12. 1969; Schauffs Gespräche mit dem venezolanischen Außenminister Calvani betr. „Zusammenarbeit mit Deutschland auf dem Wirtschafts- (und) insbesondere auf dem Erdölgebiet" am 6. 9. und 5. 10. 1970, Kopien der Gesprächsnotizen an Dr. Lögters/DEMINEX, Prof. (Horst) Ehmke, Dr. Lantzke/Wirtschaftsministerium (IfZ, NL Schauff, Bd. 39); Schreiben Gerold Moser an Schauff vom 25. 1. 1977 (IfZ, NL Schauff, Bd. 5). Zu Schauffs Diensten bei der Etablierung eines deutsch-venezolanischen Luftverkehrsabkommens vgl. den "Vermerk“ von Michael Schauff Dezember 1969 (IfZ, NL Schauff, Bd. 27). Am Jungfernflug der Lufthansa nach Venezuela nahmen Johannes und Karin Schauff zusammen mit Minister Leber und Ehefrau teil. Vgl. die Korrespondenz Johannes Schauff mit dem Public Relations Direktor der LH, Carl Wingenroth, 21. und 30. 6. 1971, mit Namensliste der Teilnehmer des Eröffnungsfluges (IfZ, NL Schauff, Bd. 38).

61 Schauff hatte 1973 den venezolanischen Orden 1. Klasse „Francisco de Miranda“ verliehen bekommen; im gleichen Jahr wurde er mit dem brasilianischen Komturkreuz des Ordens „Rio Branco“ geehrt (IfZ, NL Schauff, Bd. 27); zu Schauffs vermittelnder Rolle bei den Verbindungen der Bundesrepublik insbesondere zu Venezuela vgl. die Notizen des damaligen parlamentarischen Staatssekretärs im Bundeskanzleramt, Karl Theodor von und zu Guttenberg (BA, NL Guttenberg, Sign. 42, u.a. Bl. 101, 107). 